\title{
Designing and manufacturing of a user friendly and affordable nasal suction machine for Acute Bronchiolitis treatment of children in Oman
}

\author{
Syed Riyadul Mahi \\ Jibreal Khan \\ Fiseha Mekonnen Guangul \\ MIDDLE EAST COLLEGE \\ Middle East College \\ Middle East College
}

Acute Bronchiolitis is a disease which usually infects children of the age 0 to 6 years old. The patients infected by this disease require medical attention at hospitals. The patients must be admitted in the hospitals for a period of 2-3 days. Parents of the patients must stay in the hospital along with them. This causes lack of hospital beds, more expense of the hospital to provide extra food for the parents. By a qualitative research, it was found that parents would want an affordable device so that they could care for their children from home instead of staying at the hospital. Parents from all over Oman come to the capital so that their children can be treated. Children also tend to get exposed to more sickness by being around sick children at the hospital. Taking this problem into consideration, a circuit has been designed in such a way it was easy to use and that it could be used by parents to treat their children at home without the need to visit a hospital. The circuit works on Arduino Uno and has various pre-existing modules attached to it, the estimated cost of the device is far lesser than what the mainstream industrial devices cost. The device consists of a Bluetooth Module for the purpose of connectivity via cellphone, a $12 \mathrm{~V}$ vacuum pump to suck the mucus out of the patient's lungs, variable resistor to control the force of vacuum and a rubber tube capable of fitting into the nostril to allow suction of mucus. This simple device made from the counter parts can offer significant benefits to patients affected by Acute Bronchitis.

\section{Introduction}

Since children have a weak and developing immune system, they get prone to around six types of viral infection in a year. Depending on the intensity of the virus, the virus might affect the upper, or the lower, or both the respiratory tracts at the same time. When the lower respiratory tract is infected, the child has a probability of getting the sickness bronchiolitis (Tessini, 2018). Bronchiolitis is the term given to the disease which is provoked by a virus mainly Respiratory Syncytial Virus more commonly known as RSV, or another virus called Human Rhinovirus. It mainly affects children and infants. The bronchioles of the lungs get inflamed and swollen, causing difficulty in breathing for the child (Mayo Clinic, 2019). Bronchiolitis affects almost all the children in this world. Initially the symptoms have the same symptoms as that of a common cold which is a runny nose and the normal cough. It can be generally cured at home. The parents need to help their child to clear the upper nasal tract. As time passes by, the child will face more complication in breathing. The parent should know that it is time to take the child to the hospital when the child starts "wheezing" when he or she breathes out air. Wheezing is the term given to the sound of a whistle during exhalation. This happens due to the inflammation of the bronchioles and mucus blocking the airways. The child becomes sluggish, breathes heavy while eating or drinking and at times refusing to eat. This calls for medical attention and there is no medicinal treatment for acute bronchiolitis. There are only temporary fixes so that the child can feel good. This includes nasal and oral suctioning, oxygen therapy for the more severe cases. 


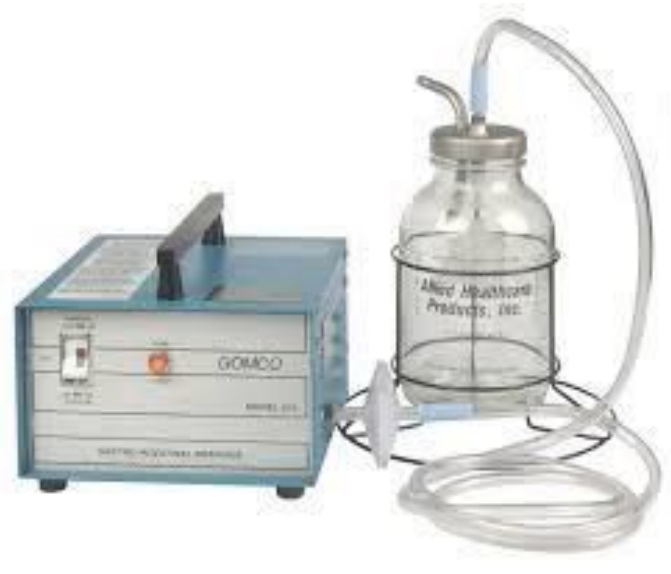

Figure 1. A hospital use nasal suction machine for the suction of mucus (source - Google images)

Unfortunately, most of the times, the children must be admitted to the hospital because suctioning is done around 3-4 times a day for the child to feel at ease. In Oman during winter, lots of children are admitted in hospitals for the treatment of acute bronchiolitis. Parents come from far and wide from all over Oman to central hospitals in the capital so that their children can receive medical attention. The portable machines available in the market are not user friendly and they are expensive. Parents tend to not purchase that device.

\section{Problem Statement}

- Children are more susceptible to various diseases when admitted to hospitals.

For acute bronchiolitis treatment, children are admitted to the hospital. They are more prone to get sick since they would be exposed to various other diseases from other patients. Children usually get admitted for a day, but when cases are severe, they get admitted for 2-3 days. Many parents unwillingly admit their children. They must stay alongside their children. This is a problem for the parents as well as the hospital management since it would be extra cost for the hospital. During winter seasons, there are shortages of bed due to the heavy influx of patients diagnosed with acute bronchiolitis. Hospitals have a difficulty in managing beds for these patients.

- Nasal aspirators available in the market are expensive and hard to maintain.

The nasal aspirators available in the market are expensive, at around OMR 50. They are not affordable for a family with average income. Hence, citizens just accept using their free of cost medical system at government hospitals. Even the aspirators used in hospitals are over OMR 1200. They are exported from a company called "medela ${ }^{\circledR ”}$ and are exported from Switzerland.

\section{Aim}

The main aim of this research was to design and manufacture of a user friendly and affordable nasal suction machine for Acute Bronchiolitis treatment. The machine that would be created could be used at home by parents without any medical assistance.

\section{Literature Review:}

Stanciu and Mucioniu (2015) have proposed a portable medical device based on Arduino which consists of a variety of sensors to monitor the patients Respiration , Blood pressure , Body Temperature and provide an Electrocardiogram. The system provides all this information in real- 
time and ends the need for separate equipment's for separate measurements.

Wu and Shieh (2016) have presented the prototype of a device capable of making ECG measurements. The device uses ECG signal sensors combined with Arduino Microcontroller. NILabView is then used as a virtualinterface to display the ECG signal. The results are then stored in MySQL database and can be viewed through other devices.

Rashid and Shekha (2017) have proposed a system capable of controlling the flow of a liquid for medical applications such as IV drips. The system is based on an Arduino microcontroller. The rate of flow of liquid is monitored using flow sensors and can be controlled via Bluetooth using an Android smartphone. Many ideas have been proposed which aim to use simple microcontrollers and affordable parts to replace expensive and bulky medical equipment. This paper hopes to build on those ideas.

\section{Hardware description and requirements:}

The system consists of an Arduino Uno Microcontroller, a $12 \mathrm{~V}$ vacuum pump , a Potentiometer , LCD , Relay , External Battery and a Bluetooth module.

The Arduino microcontroller controller switches the Vacuum pump on or off depending upon the input it receives from the user via the Bluetooth module. The Arduino is programmed using $\mathrm{C}$ language and consists of 14 I/O pins out of which 6 of them can provide PWM outputs.

The system requires the use of a device capable of suction hence a $12 \mathrm{~V}$ vacuum pump is used. The vacuum pump has 2 chambers one which sucks is air and the other which throws the air out. As the maximum voltage that can be provided by the Arduino is $5 \mathrm{v}$ an external power supply is required to operate the $12 \mathrm{~V}$ pump.

The system requires a potentiometer to control the pressure applied by the vacuum pump , as the resistance of the potentiometer changes so does the current flowing through the Vacuum thereby changing the pressure at which it sucks.

The Bluetooth module used is the HC-05. It enables the microcontroller to receive commands from a device via Bluetooth. These commands are used to turn the Pump on and off. Devices such as Smartphones can easily be connected.

The vacuum pump requires a voltage of $12 \mathrm{~V}$, as the Arduino can only provide a maximum voltage of $5 \mathrm{v}$ an external $12 \mathrm{~V}$ battery is required. Th external battery is connected to the Motor through the Normally Open side of the Relay module

A LCD is used to display details such as the Total Runtime of the pump, if the pump is ON/OFF, the number of times the pump has used and if the pump needs to be cleaned. 


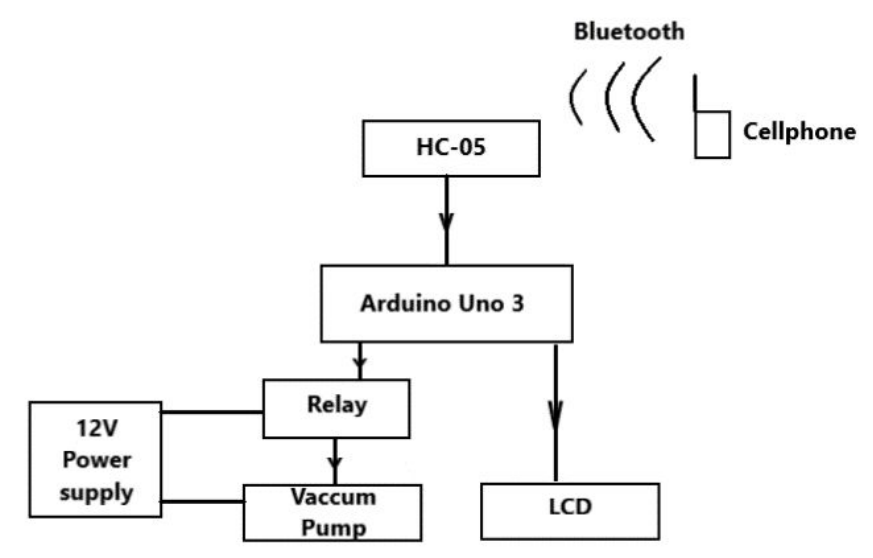

Figure 2. System Block Diagram

\section{System Analysis}

The module HC-05 provides Bluetooth connectivity to the system, it allows the system to connect with any device capable of handling Bluetooth connectivity. Once the device is paired with the system , the user can use an app from the play store specifically designed for the purpose of sending commands from an Android smart phone to the Arduino serial port via Bluetooth. Upon receiving an input from the user, the module sends the received input to the Arduino Microcontroller. Arduino then compares the input to a pre-set value and uses a series of "IF ELSE" conditions to take the appropriate step. When the input received from the user is 1 or HIGH the Arduino turns the relay connecting the external battery and the vacuum pump on thereby switching on the pump. Once the pump turns on, its pressure can be controlled through a potentiometer which controls the current flowing through the pump. The pump sucks in air through one chamber which is connected to a rubber tube and throws out air from the other chamber which too is connected to a rubber tube. The status of the pump is displayed on an LCD, this includes details such as pump running time and the suction power of the pump. Upon receiving a 0 or LOW from the user, the relay connecting the pump and the external battery is switched off by the Arduino thereby turning the pump off. The system is programmed to alert the user to clean up the pump after it has been used several times in order to maintain hygienic conditions. It displays a message on the LCD alerting the user that it is time to clean up the pump along with the count of how many times the pump has been used.

Figure 3. System Flowchart

\section{Pseudo Code:}

If (Input Received by the Bluetooth Module=high)

\{

Turn Pump=ON 


\section{Display Data On LCD Screen}

Number of times the pump is used ++

If (Number of Times the pump is used $>=15$ )

\{

Display (“Clean up Pump”)

\}

\}

else (Input Received by the Bluetooth Module=low)

\{

Turn Pump=OFF

Turn off LCD Screen

\}

\section{System Testing:}

\section{Establishing connection to the Device:}

The system consists of HC-05 Bluetooth module which is used to establish connection with the Device. Any smartphone can be connected if it uses the $2.4 \mathrm{Ghz}$ frequency range for Bluetooth communication. The device remains connected to the smartphones for up to 10 meters. In order for the smartphone to send commands to the Arduino Microcontroller it requires an application called 'Arduino Bluetooth Controller'. There are several other applications that provide similar functionality. The app consists of programmable buttons which can send alphanumeric data to the serial monitor of Arduino. This data is evaluated under a series of IF conditions and the required action is taken. If the data received by the device is 1 , it switches on the relay and thereby turns on the vacuum pump. If the data received by the device is 0 , it switches off the relay and thereby turns off the pump.

\section{Figure 4. Application Interface}

\section{Results}

The suction power of the pump can be adjusted by manually rotating the potentiometer. In order achieve the required suction power. Usually a pressure of $5.8 \mathrm{psi}$ is required in order to drain the mucus.

The suction capabilities of the pump are strong enough to suck the water from one end and disperse it through the other end. As water is similar to the density of the fluid to be extracted from the patients. 
The data such as the active time of the pump, the current status of the pump, number of times the pump has been used etc. are displayed on the LCD in the system.

The application used to interact with the system via Bluetooth is called "Arduino Bluetooth controller". The data sent from each clickable button can be altered using the app.

Figure 5. System Prototype

\section{Conclusion and Recommendations:}

The device was designed keeping in mind that patients suffering from acute bronchitis are required to frequently visit the hospitals in order to use the devices that help them breathe better. In order to dismiss the requirement of having to visit the hospital , a cheap and portable system capable of extracting mucus from a patient has been manufactured. The device uses an Arduino microcontroller which receives input from the user via Bluetooth and based on the input received switches on a vacuum pump. The vacuum pump has 2 chambers one to suck in the air and the other to push out the air. Each of these chambers is connected to a rubber tube small enough to fit in the nose pipe of the patient. The device is very easy to use. Parents just need to insert the pipe through the nasal cavity for suction. Through the click of a button of Arduino Bluetooth controller the suction begins, and it can be switched off too. The device was created with less than half the price available in the market.

The device can be made more compact by using a smaller microcontroller like the Arduino Nano. Also, a mechanism to determine the suction pressure being offered by the device can be added for better performance.

\section{Acknowledgement}

We would like to thank our supervisor Dr. Fiseha Mekonnen Guangul for his amazing guidance and support. We would also like to thank Middle East College for letting us use its facilities and giving us this opportunity.

\section{References:}

Mayo Clinic. (2019). Bronchiolitis - Symptoms and causes. [online] Available at:

https://www.mayoclinic.org/diseases-conditions/bronchiolitis/symptoms-causes/syc-20351565

[Accessed 25 Nov. 2019].

Tessini, Brenda L. 2018. Overview of Viral Respiratory Tract Infections in Children. [ONLINE] Available at:

https://www.msdmanuals.com/home/children-s-health-issues/viral-infections-in-infants-andchildren/overview-of-viral-respiratory-tract-infections-in-children. [Accessed 25 Nov. 2019].

Stanciu, L. and Mucioniu, A. (2015). Multifunctional portable equipment for medical and biometric applications. 2015 E-Health and Bioengineering Conference (EHB). [online] Available at: https://ieeexplore.ieee.org/document/7391611 [Accessed 23 Nov. 2019].

Wu, M., Shieh, S., Liao, Y. and Chen, Y. (2016). ECG Measurement System Based on Arduino and Android Devices. 2016 International Symposium on Computer, Consumer and Control (IS3C). [online] Available at: https://ieeexplore.ieee.org/document/7545287 [Accessed 25 Nov. 2019].

Rashid, H., Shekha, S., Reza, S., Ahmed, I., Newaz, Q. and Rasheduzzaman, M. (2017). A low cost 


\section{Journal of Student Research}

Fourth Middle East College Student Research Conference, Muscat, Sultanate of Oman

automated fluid control device using smart phone for medical application. 2017 International Conference on Electrical, Computer and Communication Engineering (ECCE). [online] Available at: https://ieeexplore.ieee.org/document/7913014 [Accessed 24 Nov. 2019]. 